\title{
Características psicológicas y estilos cognitivos de estudiantes de medicina y de otras carreras de la Pontificia Universidad Católica de Chile
}

\author{
Marcela Bitran Ca, Denisse Zúñiga Pb, Montserrat Lafuente G, \\ Paola Viviani G C, Beltrán Mena C. \\ Psychological features and cognitive \\ styles of students entering Medicine and \\ other careers at the Pontificia \\ Universidad Católica de Chile
}

\begin{abstract}
Background: The similarity between the psychological features of medical school freshmen of different cohorts suggests that Medicine attracts students with specific psychological types. However, it is also possible that medical students are similar to the students admitted to any other career with high admission requirements. Aim: To determine if medical school freshmen are different from those of Engineering, Architecture, Psychology and Journalism. Subjects and methods: The Spanish version of the Myers Briggs Psychological Type Indicator (MBTI) was applied to two cohorts of Medical School freshmen (90 students of the 2000 cohort and 91 students of the 2001 cohort) and to a sample of 669 freshmen from the careers of Engineering, Psychology, Architecture and Journalism. Results: Students entering Medical School are similar to the students admitted to Engineering and different from those that entered Architecture, Psychology and Journalism in the Pontificia Universidad Católica de Chile in 2000 and 2001. Medicine attracts a larger proportion of concrete and practical students that have an objective and systematic approach to study and to life in general. Unlike Medicine, Psychology and Architecture attract more students that have a cognitive style characterized by an intuitive perception, and that face life with an open and flexible attitude. Conclusions. This study reveals that the psychological features of undergraduate students are associated to their career choice. These psychological variables, therefore, may be relevant to the students' vocational preferences and possibly to their future specialty choice (Rev Méd Chile 2004; 132: 809-16).
\end{abstract}

(Key Words: Education, medical; Personality inventory; Psychological tests; Students, medical)
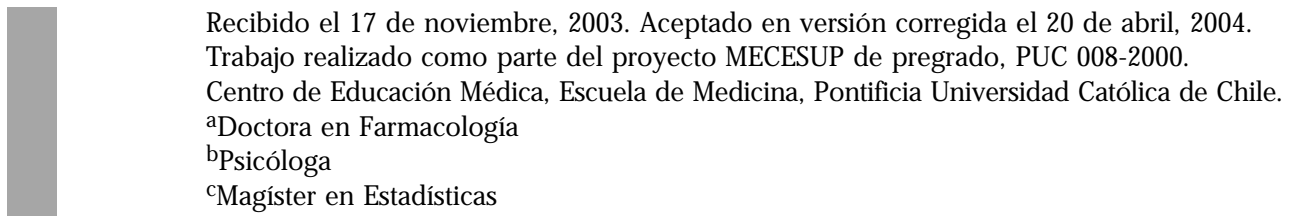

Correspondencia a: Marcela Bitran C. Centro de Educación Médica, Escuela de Medicina, Pontificia Universidad Católica de Chile. Edificio Escuela de Medicina, 6o piso. Lira 44, Santiago Centro. Fono: 354-3811. Fax: 633-1457.

E-mail: mbitran@med.puc.cl 
T a personalidad y estilo cognitivo influyen en la manera particular en que cada estudiante percibe y procesa la información durante el aprendizaje ${ }^{1}$. Por ello, diversas escuelas de medicina de Norteamérica y Europa incluyen estas variables en el portafolio de sus alumnos ${ }^{2}$. En la Escuela de Medicina de la Pontificia Universidad Católica de Chile (PUC) iniciamos en el año 2000 un estudio longitudinal denominado Psimed 21, destinado a conocer las características de personalidad y estilos cognitivos de nuestros estudiantes y su relación con el desempeño académico y aproximación al aprendizaje. Los resultados de la fase inicial del Psimed 21 mostraron que hay un patrón definido y estable en las personalidades y estilos cognitivos de los estudiantes que ingresan a medicina ${ }^{3}$. La mayoría de ellos se caracteriza por usar un discernimiento lógico y objetivo; tienen una actitud estructurada y ejecutiva ante la vida, y un estilo cognitivo centrado en la percepción de la información concreta y en el uso práctico de ésta ${ }^{3}$. En contraste, cerca de un tercio de nuestros estudiantes tiene características opuestas: privilegia en sus decisiones la consideración por los demás, la armonía interpersonal, y tiene una actitud más flexible y perceptiva ante la vida ${ }^{3}$.

La falta de información acerca de las características psicológicas y estilos cognitivos de los estudiantes de nuestro país, nos impide establecer si el perfil de nuestros alumnos es representativo de la población general de estudiantes que ingresa a la universidad. Tampoco sabemos si este perfil es propio de quienes optan por medicina 0 si es independiente de la opción vocacional.

El presente estudio intenta determinar si los alumnos de medicina tienen patrones de persona- lidad y estilos cognitivos característicos o si, por el contrario, son semejantes a estudiantes que optan por otras carreras universitarias con similares exigencias de admisión. Para ello comparamos el patrón de preferencias psicológicas y estilos cognitivos determinados con el Myers Briggs Type Inventory (MBTI) de los estudiantes admitidos a medicina, ingeniería, arquitectura, psicología y periodismo en la PUC en 2000 y 2001.

Esperamos que este estudio motive a los educadores a conocer las características de personalidad y estilos cognitivos de sus estudiantes y a poner este conocimiento al servicio de su formación profesional.

\section{MATERIALES Y MÉTODOS}

Población. Participaron en el estudio las cohortes de ingreso 2000 y 2001 de medicina de la PUC ( $n=90$ y $\mathrm{n}=91$, respectivamente). Las preferencias psicológicas y estilos cognitivos de estos estudiantes se compararon con las de jóvenes que ingresaron a ingeniería, arquitectura, psicología y periodismo en igual período. Con el objeto de minimizar las diferencias atribuibles a las exigencias de admisión, se eligieron las carreras mencionadas las que, representando campos profesionales distintos, tienen puntajes de selección comparables ( $>725$ puntos). La aplicación del test se realizó (previo consentimiento informado) en el primer semestre del primer año, a los alumnos que se encontraban presentes durante clases de gran convocatoria. Los 850 participantes corresponden a $56 \%$ de los estudiantes matriculados en 2000 y 2001 en las carreras estudiadas (Tabla 1). Las muestras constituyen entre $38 \%$ (periodismo) y 99\% (medicina) de sus poblacio-

Tabla 1. Tamaño y distribución por sexo de las poblaciones y muestras de cada carrera. Los datos corresponden a las cohortes de ingreso 2000 y 2001.

\begin{tabular}{|c|c|c|c|c|}
\hline & \multicolumn{2}{|c|}{ Población } & \multicolumn{2}{|c|}{ Muestra } \\
\hline & $\begin{array}{l}\text { Estudiantes } \\
\text { (n) }\end{array}$ & $\begin{array}{c}\text { Relación } \\
\text { hombres/mujeres } \\
(\%)\end{array}$ & $\begin{array}{l}\text { Estudiantes } \\
\text { (n) }\end{array}$ & $\begin{array}{c}\text { Relación } \\
\text { hombres/mujeres } \\
(\%)\end{array}$ \\
\hline Medicina & 183 & $50 / 50$ & 181 & $53 / 47$ \\
\hline Ingeniería & 807 & $84 / 16$ & 304 & $86 / 14$ \\
\hline Arquitectura & 194 & $49 / 51$ & 123 & $55 / 45$ \\
\hline Psicología & 168 & $22 / 78$ & 125 & $31 / 69$ \\
\hline Periodismo & 156 & $25 / 75$ & 117 & $28 / 72$ \\
\hline Total & 1.508 & $62 / 38$ & 850 & $58 / 42$ \\
\hline
\end{tabular}


nes y su distribución por sexo es similar a la de su población respectiva (Tabla 1). En todas las carreras, las preferencias psicológicas y estilos cognitivos de los alumnos admitidos en 2000 resultaron similares a las de 2001. Dada la reproducibilidad de estos patrones, en este estudio analizamos en conjunto los datos obtenidos en 2000 y 2001. Los antecedentes biodemográficos y académicos fueron proporcionados por la Dirección de Admisión y Registro Académico de la PUC 4 .

Instrumento. Las características de personalidad y estilos cognitivos se determinaron con el Indicador de Tipos Psicológicos Myers Briggs (MBTI, forma G, versión en español) ${ }^{5,6}$ que permite clasificar a las personas según sus preferencias psicológicas (Figura 1). La preferencia psicológica se define como la tendencia natural de una persona a actuar según el modo descrito en uno u otro polo de 4 dimensiones psicológicas independientes ${ }^{7,8}:$ 1) la fuente de motivación (extraversion vs introversion), 2) la percepción de información (sensing vs intuition), 3) el discernimiento (thinking vs feeling) y 4) la actitud ante la vida (judging vs perceiving) (Figura 1). Las cuatro combinaciones posibles de percepción y discernimiento se llaman estilos cognitivos y son ST (sensing más thinking), SF (sensing más feeling), NT (intuition más thinking) y NF (intuition más feeling).

La caracterización de estas dimensiones psicológicas y sus polos respectivos se origina en la Teoría de Tipos Psicológicos de Carl Jung99. El polo extraversion (E) describe la conducta de quienes derivan su motivación y energía del mundo externo en contacto con la gente y las actividades. Introversion (I) corresponde a la preferencia de personas que se motivan en su mundo interno de vivencias e ideas. Por otra parte, podemos percibir la información ya sea de manera sensing, que nos permite darnos cuenta de las cosas a través de nuestros cinco sentidos, o intuition, que nos revela patrones y posibilidades que llamamos intuiciones. De manera análoga, discernimos de acuerdo a uno de dos modos preferentes: thinking $(\mathrm{T})$, un proceso lógico orientado a una evaluación imparcial y objetiva; 0 feeling $(\mathrm{F})$, un proceso igualmente lógico que otorga a las opciones un valor personal y subjetivo. Aunque los dos modos de percepción y discernimiento compiten por nuestra atención, desde la infancia desarrollamos una preferencia 0 tendencia natural para percibir y discernir.

La cuarta dimensión del MBTI se refiere a la actitud ante la vida. Las personas que prefieren tomar las decisiones rápidamente y planificar su vida tienen preferencia judging (J). Aquellas que tienden a mantener sus opciones abiertas hasta último minuto y están dispuestas a cambiar de planes más fácilmente tienen preferencia perceiving $(\mathrm{P})$.

El MBTI es un instrumento de autorreporte válido y confiable $7,10,11$ y uno de los tests de personalidad normal más usados en orientación vocacional ${ }^{8,12}$ y educación médica ${ }^{13}$.

Estadística. Las distribuciones de las preferencias psicológicas de los estudiantes se compararon mediante el análisis de distancia basado en estadísticas de Chi cuadrado. Los puntajes de selección se compararon mediante ANOVA.

\section{RESULTADOS}

Características biodemográficas y académicas. Según sus antecedentes biodemográficos, los estudiantes que ingresaron a medicina fueron similares a los de ingeniería, arquitectura, psicolo-

\begin{tabular}{|c|c|c|c|}
\hline $\begin{array}{l}\text { Extraversion } \\
\text { (E) } \\
\text { Sensing } \\
\text { (S) } \\
\text { Thinking } \\
\text { (T) } \\
\text { Judging } \\
\text { (J) }\end{array}$ & $\stackrel{5}{\longleftarrow}$ & $\begin{array}{c}\text { ¿Cómo me motivo? } \\
\text { ¿Cómo percibo la información? } \\
\text { ¿Cómo discierno? } \\
\text { ¿Cómo me enfrento al mundo? }\end{array}$ & $\begin{array}{l}\text { Introversion } \\
\text { (I) } \\
\text { Intuition } \\
\text { (N) } \\
\text { Feeling } \\
\text { F) } \\
\text { Perceiving } \\
\text { (P) }\end{array}$ \\
\hline
\end{tabular}

FIGURA 1. Preferencias psicológicas según el MBTI. 
gía y periodismo (Tabla 2). En lo académico, los estudiantes de medicina se distinguieron de los demás por tener un puntaje de selección promedio más alto (Tabla 2).

Preferencias psicológicas. La distribución de las preferencias psicológicas por carrera se ilustra en la Tabla 3. A medicina ingresó una proporción semejante de estudiantes con preferencia extraversion (E) e introversion (I). Lo mismo ocurrió con la percepción de información (S-N). Sin embargo, en cuanto al discernimiento (T-F) y la actitud frente a la vida (J-P) la distribución fue desigual. El 68\% de los alumnos usaba el modo thinking (T), objetivo e imparcial y el 32\% restante el feeling (F), más personal y subjetivo. El 69\% de los estudiantes mostró una actitud judging, estructurada y decidida y el 31\% restante una actitud perceiving $(\mathrm{P})$ más abierta y flexible. Las preferencias psicológicas de hombres y mujeres en medicina fueron iguales, excepto en la dimensión T-F: una mayor proporción de hombres utiliza el modo thinking $(79 \% \mathrm{H}$ vs $55 \% \mathrm{M})$. La distribución de preferencias psicológicas de medicina fue similar a ingeniería y diferente de arquitectura, psicología y periodismo (Tablas 3 y 4). Lo que distinguió a

Tabla 2. Características biodemográficas y académicas de los estudiantes según carrera

\begin{tabular}{|c|c|c|c|c|c|}
\hline & $\begin{array}{c}\text { Medicina } \\
(\mathrm{n}=181)\end{array}$ & $\begin{array}{c}\text { Ingeniería } \\
(\mathrm{n}=304)\end{array}$ & $\begin{array}{l}\text { Arquitectura } \\
(\mathrm{n}=123)\end{array}$ & $\begin{array}{l}\text { Psicología } \\
(\mathrm{n}=125)\end{array}$ & $\begin{array}{c}\text { Periodismo } \\
\quad(\mathrm{n}=117)\end{array}$ \\
\hline \multicolumn{6}{|l|}{ Antecedentes biodemográficos } \\
\hline Edad (años \pm SD) & $18,8 \pm 1,1$ & $18,4 \pm 0,6$ & $18,5 \pm 0,6$ & $19,1 \pm 1,6$ & $18,8 \pm 1,0$ \\
\hline Primogénitos (\%) & 56 & 44 & 46 & 47 & 48 \\
\hline $\mathrm{n}^{\mathrm{o}}$ hermanos $(\overline{\mathrm{x}} \pm \mathrm{SD})$ & $2,1 \pm 1,2$ & $2,4 \pm 1,4$ & $2,5 \pm 1,4$ & $2,3 \pm 1,4$ & $2,6 \pm 1,9$ \\
\hline \multicolumn{6}{|l|}{ Antecedentes académicos } \\
\hline Colegio particular (\%) & 80 & 88 & 84 & 75 & 78 \\
\hline Colegio municipalizado (\%) & 17 & 10 & 12 & 18 & 14 \\
\hline Región Metropolitana (\%) & $66^{*}$ & 80 & 81 & 75 & 75 \\
\hline Puntaje selección $(\bar{x} \pm S D)$ & $767 * \pm 9$ & $745 \pm 21$ & $737 \pm 12$ & $743 \pm 14$ & $729 \pm 21$ \\
\hline
\end{tabular}

$* p<0,05$ respecto de los estudiantes de las demás carreras.

Tabla 3. Preferencias psicológicas de los estudiantes según carrera y sexo

\begin{tabular}{|c|c|c|c|c|c|c|c|c|c|c|c|c|c|c|}
\hline \multicolumn{15}{|c|}{ Preferencias psicológicas (\%) } \\
\hline & \multicolumn{2}{|c|}{ Medicina } & \multicolumn{3}{|c|}{ Ingeniería } & \multicolumn{3}{|c|}{ Arquitectura } & \multicolumn{3}{|c|}{ Psicología } & \multicolumn{3}{|c|}{ Periodismo } \\
\hline & $\mathrm{T} \mathrm{M}$ & $\mathrm{H}$ & & M & & $\mathrm{T}$ & M & $\mathrm{H}$ & & M & & $\mathrm{T}$ & M & \\
\hline $\mathrm{E}$ & 5154 & 48 & 45 & 55 & & 37 & 38 & 37 & & 44 & 36 & 46 & 46 & \\
\hline I & 4946 & 52 & 55 & 45 & 57 & $63^{\mathrm{a}}$ & 62 & 63 & 58 & 56 & 64 & 54 & 545 & 55 \\
\hline $\mathrm{S}$ & $57 \quad 53$ & 60 & 54 & 57 & 53 & 28 & 25 & 31 & 26 & 31 & 13 & 42 & 443 & 36 \\
\hline $\mathrm{N}$ & 4347 & 40 & 46 & 43 & 47 & $72^{\mathrm{a}}$ & 75 & 69 & $74^{\mathrm{a}}$ & 69 & $87 *$ & 58 & 56 & 64 \\
\hline $\mathrm{T}$ & $68^{\mathrm{a}} 55$ & $79 *$ & $76^{\mathrm{a}}$ & 48 & $81 *$ & $63^{\mathrm{a}}$ & 53 & $72 *$ & 51 & 52 & 49 & $68^{\mathrm{a}}$ & 677 & 73 \\
\hline $\mathrm{F}$ & $32 \quad 45$ & 21 & 24 & 52 & 19 & 37 & 47 & 28 & 49 & 48 & 51 & 32 & 332 & 27 \\
\hline $\mathrm{J}$ & $69^{\mathrm{a}} 74$ & 65 & $61^{\mathrm{a}}$ & 64 & 60 & 50 & 51 & 50 & 50 & 55 & 41 & 55 & 613 & 39 \\
\hline $\mathrm{P}$ & 3126 & 35 & 39 & 36 & 40 & 50 & 49 & 50 & 50 & 45 & 59 & 45 & 39 & 61 \\
\hline
\end{tabular}

$\mathrm{T}=$ todos, $\mathrm{M}=$ mujeres, $\mathrm{H}=$ hombres; ${ }^{\mathrm{a} p}<0,05$ respecto del polo opuesto de la misma dimensión ( $\mathrm{p}$ ej: $\mathrm{T}$ vs $\mathrm{F}$ ); $*_{p}<0,05$ respecto del valor correspondiente de las mujeres. 
Tabla 4. Comparación de las preferencias psicológicas entre estudiantes de las diferentes carreras

\begin{tabular}{|lllll|}
\hline & Medicina & Ingeniería & Arquitectura & Psicología \\
\hline Periodismo & S-N*, J-P* & S-N* & S-N* & S-N**, T-F** \\
Psicología & S-N**, J-P**, T-F** & S-N*** T-F*** & & \\
Arquitectura & S-N**, J-P**, E-I* & S-N***, T-F** & & \\
Ingeniería & & & & \\
\hline
\end{tabular}

${ }^{* *} p<0,001 ; *^{*}<<0,01 ; *_{p}^{*}=0,05$. Nota: Sólo se indican las dimensiones en las que existen diferencias significativas.

medicina de estas últimas carreras fue la percepción de información y la actitud frente a la vida. A medicina ingresaron proporcionalmente más estudiantes interesados en hechos y datos concretos (S) y con una actitud estructurada y decidida (J) que a arquitectura, psicología y periodismo (Tablas 3 y 4). Además, los futuros médicos fueron más extrovertidos (E) que los 'arquitectos', y más racionales y lógicos ( $\mathrm{T}$ ) que los 'psicólogos' (Tablas 3 y 4). Los estudiantes de arquitectura fueron similares a los de psicología y diferentes a los de ingeniería y periodismo; estos últimos fueron diferentes de todos los demás (Tabla 4).

$\mathrm{Al}$ estratificar por sexo (datos no mostrados) encontramos que la diferencia de actitud (J-P) entre los estudiantes de medicina y los de arquitectura se explica por una diferencia que sólo se da en las mujeres. Esto es, las mujeres 'médicos' eran más estructuradas que las 'arquitectos', pero no hubo diferencias entre los hombres de ambas carreras. En contraste, las diferencias entre 'médicos' y 'periodistas' (dimensiones S-N y J-P), son sólo explicadas por los hombres (datos no mostrados). También atribuible sólo a los varones fue la diferencia en la dimensión T-F entre medicina y psicología: los hombres 'médicos' fueron más objetivos y lógicos que los 'psicólogos'.

Estilos cognitivos. El estilo cognitivo más frecuente entre los estudiantes que ingresaron a medicina fue el ST, que combina las funciones cognitivas sensing y thinking. Este estilo, denominado 'práctico y concreto' predominó también entre los estudiantes que ingresaron a ingeniería (Tabla 5). En arquitectura y periodismo en cambio, el estilo NT, también llamado 'lógico e ingenioso', fue el más frecuente. Psicología fue un caso especial pues hubo dos estilos igualmente frecuentes: NT y
Tabla 5. Estilos cognitivos predominantes de los estudiantes según carrera

\begin{tabular}{|lcc|}
\hline & $\mathrm{S}$ & $\mathrm{N}$ \\
$\mathrm{T}$ & Medicina & Arquitectura \\
& $(43 \%)$ & $(43 \%)$ \\
& Ingeniería & Periodismo \\
& $(44 \%)$ & $(37 \%)$ \\
& & Psicología \\
& & $(37 \%)$ \\
& & Psicología \\
$\mathrm{F}$ & & $(38 \%)$ \\
\hline
\end{tabular}

NF. NT fue más frecuente entre las mujeres y NF, denominado 'entusiasta y perceptivo' entre los hombres.

\section{Discusión}

Las preferencias psicológicas y los estilos cognitivos de los jóvenes que optan por medicina no se diferencian de los de ingeniería, pero son distintos de los que escogen arquitectura, psicología 0 periodismo. Las diferencias más importantes tienen que ver con la percepción de la información, la postura frente a la vida y el estilo cognitivo.

A medicina e ingeniería ingresan principalmente estudiantes concretos y estructurados que enfrentan los estudios con un estilo eminentemente práctico y lógico. Estos jóvenes se interesan por hechos verificables que evalúan y usan de manera objetiva e imparcial ${ }^{7,8}$. De acuerdo a estudios realizados en Estados Unidos, los jóvenes con preferencias sensing y judging y con 
estilo ST suelen ser prácticos y positivistas y ejercen profesionalmente en áreas más bien técnicas, en contacto con hechos y objetos, como la economía, derecho, cirugía y contabilidad ${ }^{7,8}$.

A diferencia de los estudiantes de medicina, los de psicología y arquitectura son más flexibles y su estilo cognitivo está marcado por la percepción intuitiva (NT y NF). Las personas con estilo NT son lógicas e ingeniosas y tienen gran capacidad para resolver problemas en áreas como las matemáticas y la computación ${ }^{7,8}$. Además, el estilo NT es el más frecuente entre los investigadores científicos ${ }^{7,8}$. Por otra parte, las personas con estilo NF son cálidas y se interesan en las posibilidades de proyectos nuevos. Suelen encontrarse en educación, psicología clínica, psiquiatría y literatura, donde despliegan su creatividad para satisfacer las necesidades humanas ${ }^{7,8}$.

Por haberse realizado con jóvenes recién ingresados a la universidad, las diferencias aquí encontradas no derivan del hecho de estudiar o ejercer una $\mathrm{u}$ otra carrera. Tampoco pueden atribuirse a diferencias académicas o biodemográficas preexistentes, pues los perfiles de ingreso de los jóvenes son muy semejantes. Pareciera más bien que las distintas carreras atraen a ciertos tipos específicos de estudiantes. La idea que ciertos individuos se sientan atraídos por determinadas actividades y se autoseleccionen para ellas, ha sido planteada tanto a partir de la teoría como de resultados experimentales ${ }^{14}$. El atractivo intrínseco de cualquier trabajo o profesión (a diferencia de atractivos extrínsecos como el dinero o el status) residiría en la oportunidad que éste brinda para usar las preferencias psicológicas o procesos mentales que dominamos mejor ${ }^{8,15}$.

Nuestros resultados son coincidentes con diversos estudios que muestran que la dimensión psicológica más frecuentemente asociada a la elección de carrera es la percepción de información $(\mathrm{S}-\mathrm{N})^{8}$. Los tipos sensoriales se interesan por carreras que, como ingeniería y medicina, tienen actividades organizadas y sistemáticas donde está muy claro el plan de trabajo a seguir y existe un feedback constante con la realidad ${ }^{8,16}$. Los tipos intuitivos, en cambio, prefieren actividades que les permitan innovar y estar siempre abiertos a nuevas posibilidades ${ }^{8,16}$.
Que los jóvenes prácticos, lógicos y estructurados prefieran medicina o ingeniería, y los más intuitivos y flexibles arquitectura y psicología no parece sorprendente ${ }^{16}$. El aporte distintivo de este estudio es que, no sólo valida científicamente estas impresiones del 'sentido común', sino que entrega nuevos elementos para comprender el proceso de aprendizaje. Pensamos que las diferencias psicológicas entre los estudiantes de una carrera 0 entre distintos profesionales de un equipo de trabajo, lejos de constituir una fuente inevitable de conflicto, pueden usarse para optimizar el aprendizaje, el desempeño y la satisfacción profesional de individuos y equipos. Sin ir más lejos, podemos suponer que en un equipo de salud cualquiera, es probable que el médico y el psicólogo tengan estilos cognitivos opuestos; el médico concreto y práctico (ST) y el psicólogo entusiasta y perceptivo (NF). Sin dudas, el entendimiento entre estos profesionales se verá favorecido por el conocimiento y respeto que éstos tengan por las diferencias individuales. Esta comprensión y respeto es particularmente importante en la formación de los médicos, pues éstos se desempeñan en ámbitos laborales cada vez más diversos y complejos que requieren de la capacidad de comunicarse con pacientes distintos y con profesionales de variadas disciplinas.

Pensamos que la información relativa a los tipos psicológicos no debiera utilizarse con fines discriminatorios en la admisión a la universidad. Ello no sólo sería cuestionable éticamente sino que implicaría desconocer el hecho que cada disciplina tiene múltiples ámbitos que requieren de los más variados perfiles psicológicos. Más aún, no sería sorprendente que las innovaciones y desarrollos impensados en una disciplina sean hechos por individuos cuyos tipos y estilos son minoritarios porque pueden mirar lo mismo con ojos diferentes.

Reconocemos que el presente estudio tiene ciertas limitaciones. La falta de información acenca de las características psicológicas y estilos cognitivos de los jóvenes que ingresan a las universidades chilenas o de los que egresan de la Enseñanza Media, no permite determinar si nuestros hallazgos son representativos. Sin embargo, diversos hechos sugieren que éstos son reproducibles y generalizables. En favor de la reproducibilidad está el hecho que, en todas las carreras estudiadas, los patrones 
de preferencias psicológicas de cohortes sucesivas de estudiantes son similares. En el caso de medicina, hemos observado esta semejanza en 3 cohortes de ingreso ${ }^{3}$. Además, el patrón psicológico de los estudiantes de medicina de la PUC es muy similar al de estudiantes de diversas escuelas de medicina norteamericanas $7,8,13,15$, e idéntico al de estudiantes del King's College School of Medicine de Inglaterra ${ }^{17}$. Estos hallazgos confirman la teoría jungiana que postula que las preferencias psicológicas son universales y no estarían determinadas por contextos culturales 9 .

\section{REFERENCIAS}

1. Newble D, Entwistie N. Learning styles and approaches: implications for medical education. Med Educ 1986; 20: 162-75.

2. LeSTER W. Assessing the Psychological Types of Specialists to assist students in career choice. Acad Med 1995; 70: 932-3.

3. Bitran M, Zúñiga D, Lafuente M Viviani, P, Mena B. Tipos psicológicos y estilos de aprendizaje de los estudiantes que ingresan a medicina en la Pontificia Universidad Católica de Chile. Rev Méd Chile 2003; 131: 1067-78.

4. Informe: Dirección de Admisión y Registros Académicos (DARA). Pontificia Universidad Católica (PUC). Estudio comparativo entre la Pontificia Universidad Católica de Chile y las demás instituciones de educación superior adscritas al Honorable Consejo de Rectores 2001, Marzo 2001; 22, 38 y 59.

5. Briggs Myers I. MBTI: Inventario Tipológico Forma G. Manual. Madrid: TEA Ediciones. 1991.

6. InCLAN A. The development of the Spanish version of the Myers Briggs Type Indicator, Form G. Journal of Psychological Type 1986; 11: 35-46.

7. Myers I, MacCAuley M, Quenk N, Hammer A. MBTI Manual: A guide to the development and use of de Myers-Briggs Type Indicator. Palo Alto, California: Consulting Psychologist Press, Inc. 1998.

8. Briggs I, Myers P. Gifts Differing: Understanding Personality Type. Palo Alto, California. Davis Black Publishing, 1995.

9. Jung C. Psychological Types. R.F.C. Hull, trans., Collected Works of C.G. Jung, Vol. 6 Bollingen Series XX, Princeton University Press 1977.
En resumen, este estudio demuestra que, en cuanto a sus características de personalidad y estilos cognitivos, los estudiantes que ingresan a medicina son similares a los de ingeniería y diferentes a los que optan por arquitectura, psicología o periodismo. Pensamos que este hallazgo es de importancia tanto para los estudiantes como para los docentes y autoridades educacionales, pues revela que la personalidad puede ser un factor importante en la elección vocacional $y$, probablemente, en la futura especialización profesional.

10. Capraro R, Capraro M. Myers-Briggs Type Indicator: score reliability across studies: a meta- analytic reliability generalization study. Educacional and Psychological Measurement 2002; 62: 590602.

11. Pittenger D. The utility of the Myers Briggs Type Indicator. Review of Educational Research 1993; 63: 467-88.

12. Martin C. Looking at Type and Careers. Palo Alto, California: Center for Applications of Psychological Type, 1995.

13. WaшcK M, Cambre K. Personality Type in Academic Medicine. J La State Med Soc 1999; 151: 37882.

14. Thomas A, Benne M, Marr M, Thomas E, Hume R. The evidence remains stable: The MBTI predicts attraction and attrition in an Engineering Program. Journal of Psychological Type 2000; 55: 35-42.

15. Stilwell N, Wauck M, Thal S, Burleson J. MyersBriggs type and medical specialty choice: a new look at an old question. Teach Learn Med 2000; 12: 14-20.

16. Ditiberio J, Hammer A. Introduction to type in College. Palo Alto, California: Consulting Psychologists Press, Inc. 1993.

17. Clack G, HeAd J. The personality 'types' of medical students and the implications for medical education. Med Teach 1998; 20: 57.

Agradecimientos

Agradecemos a las autoridades y a los estudiantes de las Escuelas de Ingeniería, Arquitectura, Psicología y Periodismo de la PUC, que participaron en este estudio, por la colaboración prestada en esta investigación. 